\title{
Pitch and duration as auditory cues to identify Japanese long vowels for Japanese learners
}

\author{
C. T. Justine Hui ${ }^{1, *}$ and Takayuki Arai ${ }^{2, \dagger}$ \\ ${ }^{1}$ Department of Mechanical Engineering, University of Auckland, 26 Symonds Street, Auckland 1010, New Zealand \\ ${ }^{2}$ Department of Information and Communication Sciences, Sophia University, \\ 7-1 Kioi-cho, Chiyoda-ku, Tokyo, 102-8554 Japan
}

(Received 10 February 2020, Accepted for publication 13 April 2020)

Keywords: Long vowel, Speech perception, Second language acquisition PACS number: 43.71.-k, 43.71.Es [doi:10.1250/ast.41.796]

\section{Introduction}

Japanese distinguishes between long and short vowels, where duration is an obvious auditory cue to distinguish between the two. Using a spontaneous speech corpus, Arai [1] reported that the ratio between average durations of long and short vowels was smaller than previously reported ratio obtained from read speech. Minagawa [2] investigated the differences in durations of Japanese long/short vowels in different pitch-accent, syllable positions and speaking rates, and found faster speaking rate to affect long vowel duration, as well as phrase-final vowel lengthening. This is supported by Hirata's observation [3] on how the absolute duration differences being greater in normal and fast speed than in slow speaking rate. These studies show that there is a great variation on the absolute length of long vowels in Japanese depending on the speaking environment, and suggest that duration to be an unreliable cue to distinguish between long and short vowels in spontaneous speech. In addition to duration, previous studies have concluded that pitch contour is used by native speakers to identify vowel quantities e.g. [4,5]. By using nonsense words with Japanese pitch patterns and a duration continuum, Kinoshita [4] established that native speakers use duration as their primary cue to differentiate between vowel quantities, and pitch as their secondary cue when the duration becomes ambiguous.

On the other hand, Japanese learners have trouble differentiating between long and short vowels [6]. Takiguchi explored how Japanese learners compare with native Japanese speakers in terms of their categorical perception boundaries in separating a long vowel from a short vowel [5]. She found that while the distinction was particularly hard for learners with native languages that do not have pitch differences, the perceptual boundary for advanced learners and learners with a tonal native language such as Mandarin became similar to that of a native Japanese speaker. In a different study, Hui found some differences between elderly and young native Japanese listeners on their use of pitch cues to distinguish between vowel quantities [7], especially when the elderly listeners have difficulties in pure tone discrimination.

\footnotetext{
*e-mail: justine.hui@auckland.ac.nz

†e-mail: arai@sophia.ac.jp
}

\subsection{Aim and motivation}

As shown by the various studies where duration varies depending on speaking environment, there may be a need to focus on the role of pitch contours to distinguish vowel length in real words, especially for Japanese learners. While previous investigations have focused on using nonsense words to examine learners' categorical boundaries e.g. [5], it may be more intuitive to use meaningful words to investigate how pitch and duration cues are used to distinguish between vowel quantities. As the words in the previous studies did not hold any meaning, the participants may have only responded to the duration cues when asked whether or not a vowel is short or long, neglecting the effect from the pitch cue.

The current study explores how duration and pitch are being utilised in a semantic context for learners of Japanese by using meaningful target words. As reported by Takiguchi, where native language and length of Japanese learning affected their durational boundaries [5], we will also investigate the effect of native language and length of learning, as well as the length of time the participant has lived in Japan and their Japanese level according to the Japanese Language Proficiency Test (JLPT). In doing so, this study aims to provide insights from a second language acquisition point of view for teaching Japanese to non-native speakers on their perception of Japanese long vowel distinction.

\section{Methodology}

The following procedure and stimuli manipulation are identical to the test design detailed in [7]. The minimal pair "obaasan" (grandmother) and "obasan" (aunt) were chosen as stimuli for this study as they differ in the length of /a/ and both words are expected to be taught at a similarly early stage for Japanese learners. Recordings were made by a native speaker from the Tokyo region speaking standard Japanese in a sound treated room with a sound recorder (Marantz PMD 671). The following sentences were recorded: "areha obasan/ obaasan desu" (It is [my] grandmother/aunt over there.) The minimal pair was put into a carrier sentence to avoid effects of speech rate, allowing listeners to gauge the relative duration for long and short vowels. The experiment has been approved by the ethics committee at Sophia University.

2.1. Stimuli manipulation

The words "obasan/obaasan" within the sentence pair 


\begin{tabular}{|c|c|c|c|c|c|}
\hline $\begin{array}{c}\text { Obasan } \\
\text { (aunt) }\end{array}$ & D1(a) & D2(a) & D3(a) & $\mathrm{D} 4(\mathrm{a})$ & D5(a) \\
\hline P1(a) & P1D1(a) & P1D2(a) & P1D3(a) & P1D4(a) & $\mathrm{P} 1 \mathrm{~g})$ \\
\hline P2(a) & P2D1(a) & P2D2(a) & P2D3(a) & P2D4(a) & P2(g) \\
\hline P3(a) & P3D1(g) & P3D2(g) & P3D3(g) & P3D4(g) & P3(g) \\
\hline P4(a) & P4D1(g) & P4D2(g) & P4D3(g) & P4D4(g) & P4(g) \\
\hline DO(g) & D1(g) & D2(g) & D3(g) & $\mathrm{D} 4(\mathrm{~g})$ & $\begin{array}{c}\text { Obaasan } \\
\text { (grandmother) }\end{array}$ \\
\hline
\end{tabular}

Duration

Fig. 1 Chart of stimuli organisation (taken from [7]).

were manipulated using Praat [8] where the duration, pitch or both were modified. Referring to Fig. 1, we have the original obasan/obaasan at the two diagonal ends of the grid. The "a" in the bracket indicates that the stimulus was manipulated from the "obasan(aunt)" original sound file, and the "g" indicates that the stimulus was manipulated from the "obaasan(grandmother)" original file.

Horizontally, we have the duration manipulation, where the length of /a/ was increased in five steps to become the same duration in the /a/ in "obaasan" and vice versa. The /a/ in "obasan" was measured to be $100 \mathrm{~ms}$ and the /a/ in "obaasan" was $250 \mathrm{~ms}$. Therefore each step increased or decreased by $30 \mathrm{~ms}$. Vertically, the pitch is modified. The correct pitch accent for "obasan" is LHHH and LHLLL for "obaasan," which can be observed from the original files, where the pitch contour lies flat between "oba-" and "-san," while in "obaasan," there is a decreasing slope in pitch in "-asan." This is indicated in Fig. 2 where the top and the bottom line represents "obasan" and "obaasan" respectively. Again, five step continual stimuli were created to match the pitch contour of "obasan" to "obaasan" and vice versa. Instead of using $\mathrm{Hz}$ as the steps, the unit of cent was used to create the pitch continuum to allow comparison between duration and pitch in a linear way. The schematic diagram in Fig. 2 illustrates the pitch contour manipulation between "obasan" and "obaasan." The pitch of the original files were normalised prior to the manipulation to limit the variation in the sound files. To make the sentence sounds more natural, the "desu" was also modified in terms of pitch accordingly. The audio files were also normalised in terms of their intensity to avoid effect of loudness on the perception of the words. The same stimuli were used in [7].

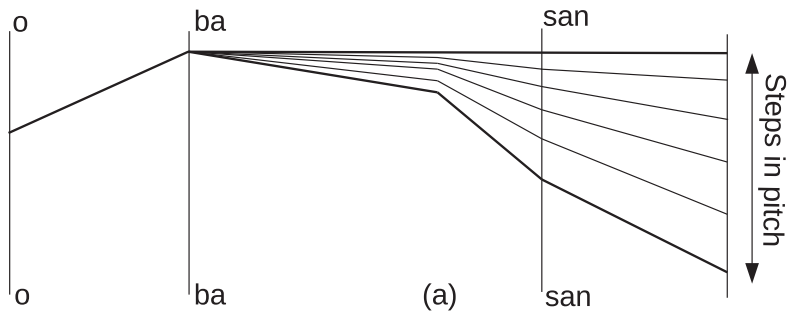

Fig. 2 Schematic representation of the pitch manipulation of stimuli (not to scale) [7].

\subsection{Test procedure}

The test was carried out in a sound-treated room and in the form of a web survey where participants accessed the survey via their own computer and headphones in a quiet environment. The purpose of utilising a web survey is to capture participants who have not lived in Japan. In the first section, the participants answered questions regarding whether or not they have lived in Japan, how long they have lived in Japan, where in Japan they have lived the longest, how long they have studied Japanese for, their native languages, and their Japanese ability via the Japanese Language Proficiency Test (JLPT).

The participants then listened to the 36 tokens through headphones in random order and answered the following question: "Who is it over there?," by choosing either "Grandmother" or "Aunt." The answer was provided in English to focus on the semantics of the word and to avoid effect from the orthographic form in Japanese, where grandmother has an extra "a" and aunt does not. For non English native participants, the meanings of the words were confirmed with them prior to the test. Participants recruited for the web survey listened to each token three times and participants who came to the sound treated room listened to each token six times.

\section{Results}

Thirty nine Japanese learners participated in the test, where twenty of them completed the web survey, and nineteen were invited to the sound treated room. No significant difference was found between the two groups and therefore the results were combined for analysis. Fifteen native speakers from [7] were included as a control group to show how native speakers behave compared to their non-native peers. Figures 3 and 4 show the average results in terms of the pitch continuum. The figures show that while there was a clearer cut boundary when duration was ambiguous $(D=2)$ for native speakers, pitch cues were still being used by non-native speakers.

\subsection{Effect of length of stay and study}

The biggest effect we expected on the perception of long vowels was whether or not the participant has lived in Japan or not, and the length of time they have lived in Japan. Following this, we also expected an effect of the length of study on their interpretation on the words.

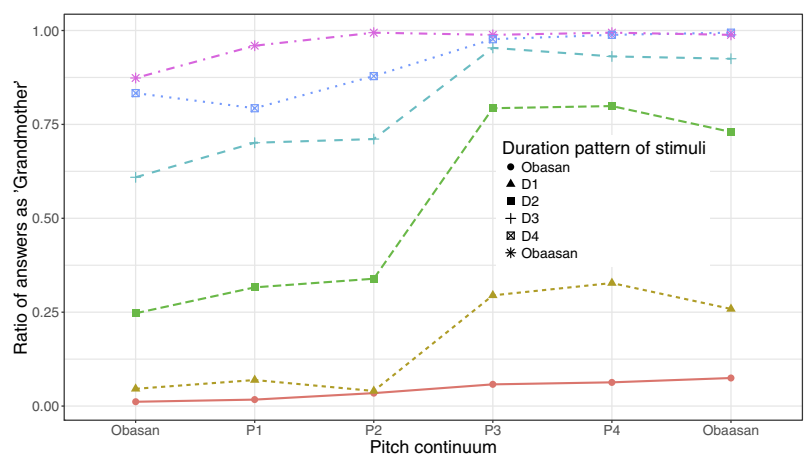

Fig. 3 Averaged results of non-native learners. 


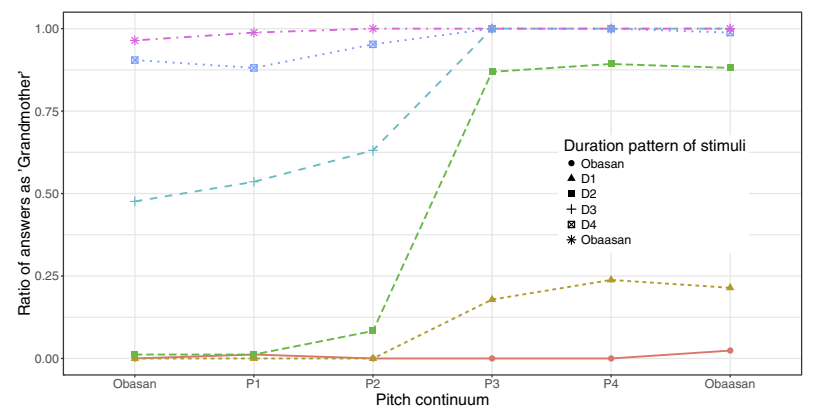

Fig. 4 Averaged results of native speakers.

The length of stay and study were categorised into four to five groups according to the duration (in parenthesis are the number of participants): never lived in Japan (10), 0-1 years (20), 1-2 years (6) and 2-5 years (3) for length of stay, and $0-1$ years (18), 1-2 years (7), 2-5 years (16), 5-10 years (6) and 10-20 years (7) for length of study, where the number within the bracket represents the number of participants.

We performed a logistic mixed effects analysis using $\mathrm{R}$ [9] and lme4 [10] to examine the effect of length of stay and length of study. Living in Japan is defined to be learners who have stayed in Japan for over one month at the time of the experiment. As fixed effects, we entered in whether the participant has lived in Japan or not, the pitch level and the duration level into the model. As random effect, the participant ID was included in the model. Using a likelihood ratio test, we found no evidence of significant difference between learners who have lived in Japan and those who have not $\left(\chi^{2}(1)=0.97, p=0.33\right)$. We then investigated the length of stay by including the factor as one of the fixed effects, and again the participants as the random effect, and found no evidence of any significant effect $\left(\chi^{2}(1)=0.012, p=0.91\right)$. Similarly, for length of studying Japanese, we found no effect from length of study on the perception of "aunt" and "grandmother" $\left(\chi^{2}(1)=0.05, p=0.83\right)$.

3.2. Regions resided

Due to the different influences of dialects around Japan, we also took into consideration the place the Japanese learners have stayed the longest. The majority of participants have lived in Tokyo region (18), which includes both Saitama and Kanagawa, followed by Osaka (4) and Kyoto (4), and one participant each for Gumma, Hyogo, and Fukuoka. Applying a logistic mixed model analysis, we found a slight effect of the regions on the results $\left(\chi^{2}(5)=13.5, p=0.019\right)$. Looking into it further by carrying out a Tukey post-hoc test, there is a significant difference between the results from participants who have resided in Kyoto and Fukuoka $(z=-3.08, p=$ $0.022)$ and between Fukuoka and Gunma $(z=-2.93, p=$ $0.03)$. However, as there was only one participant from Fukuoka, we cannot make any conclusion from the current dataset.

3.3. Native language of participant

Almost half of the participants were native English speakers (19), followed by native speakers of Mandarin (10) and Cantonese (3). The remaining participants were native speakers of Vietnamese (2), Bulgarian (1), Russian (1),
German (1) and Azerbaijani-Persian (1). Takiguchi (2011) has found an effect from the native languages of the learners on their use of pitch cues to identify long vowels [5]. However, the current study, using a logistic mixed effect model analysis with the native language as the fixed effect showed that there was no evidence of significant difference between the different native languages $\left(\chi^{2}(8)=4.73, p=0.79\right)$.

We also separated the participants according to their native language being tonal or not, where tonal languages include Mandarin, Cantonese and Vietnamese, and the remaining languages considered to be non-tonal. Again, no significant difference was observed between the two types of languages $\left(\chi^{2}(2)=0.24, p=0.89\right)$.

\subsection{Japanese Language Proficiency Test}

The Japanese Language Proficiency Test is offered worldwide and evaluates non-native Japanese learners' ability of the language. Twenty four of the learners have passed the test, with 10 having passed N1 (highest), 5 for N2, 4 for N3, 4 for N4 and 1 for N5. Applying a logistic mixed model analysis on the effect of JLPT level yields no significant difference amongst the different levels $\left(\chi^{2}(4)=4.12, p=0.39\right)$.

\section{Discussion}

We expected Japanese learners with little experience in Japan to not be able to use the pitch cues. However, looking at Fig. 3, we can clearly see that once the duration was ambiguous, the answers were motivated by the pitch cues, regardless of the length of stay, length of study, native language of the participant or their Japanese ability. This result contradicts our expectation, potentially suggesting a more innate universal approach for when listeners encounter an ambiguous word despite their experience with the language and they would use the remaining cue to distinguish the words.

Examining the stimuli where duration is ambiguous however, we were able to separate the learners into three distinct group: where the category boundary resembles native speakers as shown in Fig. 5, where there is a less vigorous slope as in Fig. 6, and lastly, where the response was horizontally flat in Fig. 7. We found a significant difference between the native-like group and the group with the flat boundary $(z=-2.51, p=0.03)$. This shows that while some learners are able to use pitch similar to their native counter-

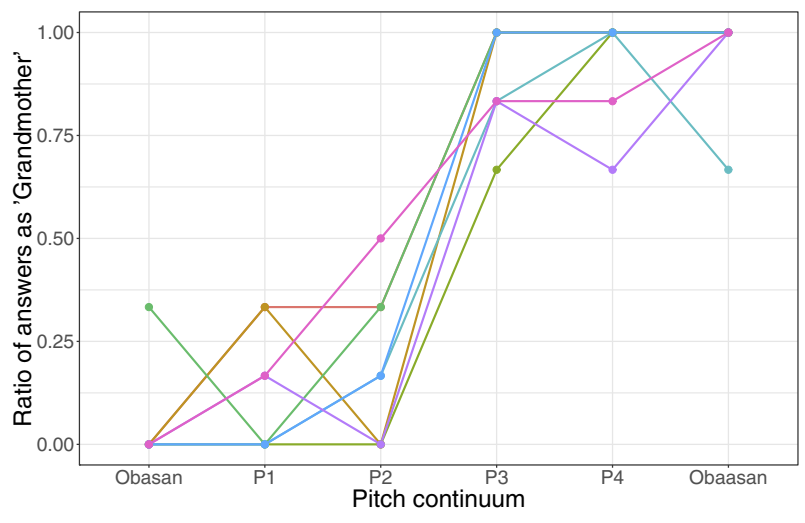

Fig. 5 Japanese learners with a native like response. 


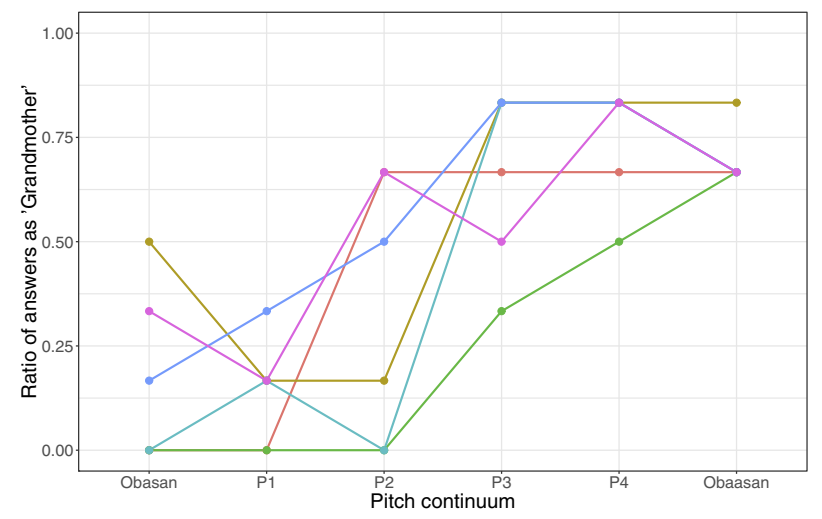

Fig. 6 Japanese learners with a pitch influenced response.

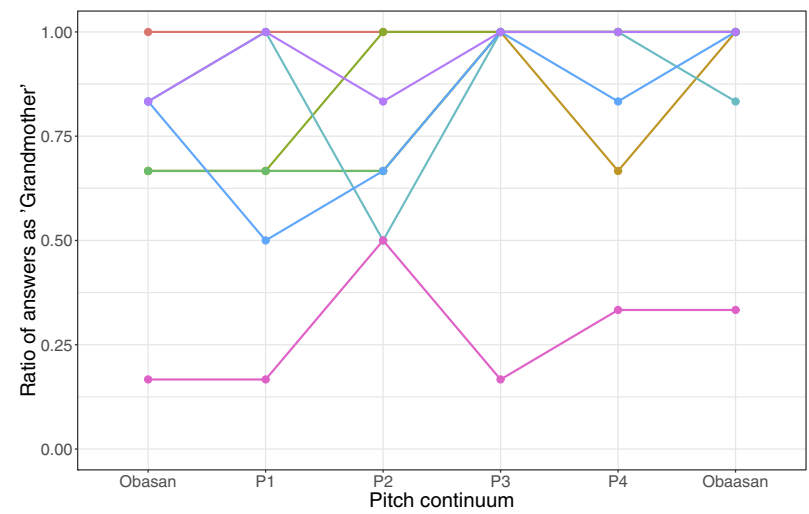

Fig. 7 Japanese learners with a flat response.

parts, there were learners who did not realise pitch cues were available. However, we were not able to explain the groupings by any of the information we have regarding the participants. We observed that the participants in the last group seem to have a longer exposure to Japanese, while having very little experience living in Japan. But again, no significant effect was found between the interaction of length of study and length of stay $\left(\chi^{2}(1)=0.05, p=0.82\right)$. Further experiment with more participants will be needed to verify this observation.

\section{Conclusion}

The current study examines how pitch and duration affect Japanese learners in distinguishing Japanese long and short vowels. We found that whether or not the learner has lived in Japan, their length of stay in Japan, length of studying Japanese, their native language, their Japanese ability measured through the Japanese Language Proficiency Test have no effect on their perception when deciding between the stimuli pair "obasan" and "obaasan." However, we also observed a possible pattern when the duration of the stimulus became ambiguous, some participants knew to use pitch, while others did not. As this observation cannot be explained by the current study, further investigation is needed.

\section{Acknowledgement}

We would like to thank our participants and Dr. Keiichi Tajima and Dr. Masako Fujimoto for their comments on the initial test design.

\section{References}

[1] T. Arai, N. Warner and S. Greenberg, "Analysis of spontaneous Japanese in a multi-language telephone-speech corpus," Acoust. Sci. \& Tech., 28, 46-48 (2007).

[2] Y. Minagawa, T. Kagomiya and K. Maekawa, "Durational variations of Japanese long/short vowels in different speaking rates: Analysis of a spontaneous speech corpus," Proc. Int. Congr. Phonetic Sciences, pp. 2127-2130 (2003).

[3] Y. Hirata, "Effects of speaking rate on the vowel length distinction in Japanese," J. Phon., 32, 565-589 (2004).

[4] K. Kinoshita, D. M. Behne and T. Arai, "Duration and F0 as perceptual cues to Japanese vowel quantity," Proc. 7th Int. Conf. Spoken Language Processing, pp. 757-760 (2002).

[5] I. Takiguchi, "Perceptual development on the identification of length in L2 Japanese," Proc. Int. Congr. Phonetic Sciences, pp. 1950-1953 (2011).

[6] K. Tajima, H. Kato, A. Rothwell, R. Akahane-Yamada and K. G. Munhall, "Training English listeners to perceive phonemic length contrasts in Japanese," J. Acoust. Soc. Am., 123, 397-413 (2008).

[7] C. T. J. Hui and T. Arai, "Elderly listeners' identification of Japanese long vowel pair 'obasan' and 'obaasan' using pitch and duration,” Acoust. Sci. \& Tech., 40, 105-115 (2019).

[8] P. Boersma and D. Weenink, Praat: doing phonetics by computer, Available at http://www.fon.hum.uva.nl/praat/ (2016) (accessed 7 Aug. 2020).

[9] R Core Team, R: A language and environment for statistical computer (2015).

[10] D. Bates, M. Mächler, B. M. Bolker and S. C. Walker, "Fitting linear mixed-effects models using lme4,” J. Stat. Softw., 67, 148 (2015). 